\title{
Scanner Device
}

National Cancer Institute

\section{Source}

National Cancer Institute. Scanner Device. NCI Thesaurus. Code C50158.

An electronic device that generates a digital representation of an image for data input to a computer or a receiver designed to search for a signal within a specified frequency range. 